\title{
The Use of Adenosine in Cerebral Aneurysm Clipping: A Review
}

\author{
Shariq A. Khan - Shahid M. Nimjee • \\ Nicole N. Guinn • Ali R. Zomorodi • \\ Arthur M. Lam - Gavin W. Britz · David L. McDonagh
}

Published online: 21 May 2013

(C) Springer Science+Business Media New York 2013

\begin{abstract}
Management of intracranial aneurysms continues to evolve, with coiling of aneurysms becoming an increasingly used modality. However, for aneurysms which are considered to be "complex" due to their size, position, or morphology, clipping continues to be the preferred treatment option. Several techniques can be utilized intraoperatively to facilitate aneurysm exposure and clip ligation. These include deep hypothermic circulatory arrest, endovascular balloon occlusion with suction, and, most commonly, temporary clip application to the proximal feeding vessel. The use of these techniques has been limited by the associated significant morbidity and mortality, and in the case of temporary clipping, anatomic limitations preventing clip application in certain cases. Recently, adenosine induced transient circulatory arrest has undergone a resurgence in neurosurgical practice to assist in complex aneurysm clip ligation. In this article we review
\end{abstract}

Electronic supplementary material The online version of this article (doi:10.1007/s40140-013-0018-5) contains supplementary material, which is available to authorized users.

S. A. Khan · N. N. Guinn · D. L. McDonagh $(\bowtie)$

Department of Anesthesiology, Duke University Medical Center,

DUMC Box 3094, Durham, NC 27710, USA

e-mail: david.mcdonagh@duke.edu

S. M. Nimjee · A. R. Zomorodi

Departments of Surgery (Neurosurgery) and Radiology,

Duke University Medical Center, Durham, NC, USA

A. M. Lam

Neuroscience Institute, Swedish Medical Center, Seattle,

WA, USA

G. W. Britz

Department of Neurosurgery, Methodist Hospital, Houston,

TX, USA this technique with special emphasis on appropriate patient selection and safety profile.

Keywords Adenosine - Cardiac arrest - Cerebral aneurysm $\cdot$ Craniotomy $\cdot$ Neuroanesthesia

\section{Introduction}

The prevalence of intracranial aneurysms is $5 \%$ in the general population [1]. The annual rate of aneurysmal rupture is approximately 6-8 per 100,000 (around 30,000 per year in the United states alone) [2]. Although coiling of intracranial aneurysms has grown in popularity in the past few years, open craniotomy and clip ligation continues to remain a commonly performed treatment modality. Open treatment of cerebral aneurysms usually involves initial placement of a temporary clip across a proximal feeding artery in order to decrease intra-aneurysmal turgor, thereby facilitating permanent clip placement. Certain intracranial aneurysm locations, such as a paraclinoid aneurysm, are difficult to treat with open clip ligation due to an inability to expose an anatomically suitable site for temporary clip placement [3, 4]. In these cases, innovative and novel techniques like deep hypothermic circulatory arrest, extracranial to intracranial bypass or endovascular balloon occlusion with suction have been employed to clip ligate the intracranial aneurysm [5-7]. Disadvantages of these techniques include a need for significant logistical support and the prevalence of complications like dissection of friable arteries, cerebro-embolic events and coagulopathy-associated intracranial hematomas [5].

Adenosine is an endogenous purine nucleoside, which comprises a molecule of adenine attached to a ribose sugar (ribofuranose) moiety via a $\beta-\mathrm{N}_{9}$-glycosidic bond [8]. 
Adenosine is essential for several biological processes including intracellular signal transduction (via cyclic adenosine-monophosphate) and energy transfer (via adenosine-triphosphate and adenosine-diphosphate). It also serves a function as an inhibitory neurotransmitter in the central nervous system, playing a role in sleep and arousal [8]. Many of the biological effects of adenosine (including those effecting sleep) are mediated via its action on adenosine receptors located in various tissues [9]. The effect of intravenously administered adenosine on animal hearts has been known since the early 20th century. In 1930, Honey et al. [10] studied the effects of intravenous adenosine on the human atrioventricular node, but it was not until 1955 that adenosine (as adenosine triphosphate) was used to terminate paroxysmal supraventricular tachycardia (PSVT). The FDA first approved the use of adenosine for treatment of PSVT in 1989, and within few years it replaced verapamil as the drug of choice for this indication [11].

The use of intravenous adenosine infusion (as Adenosine triphosphate) to facilitate hypotensive anesthesia in humans was first reported in 1982 [12]. Two years later, Sollevi et al. [13] were able to demonstrate that adenosine administered as an infusion of ATP was an easily titratable drug to induce hypotensive anesthesia during cerebral aneurysm surgery by virtue of its short half-life (10-20 s). Adenosine induced flow arrest was first described by Groff et al. [14•] to assist clipping of a basilar artery aneurysm. Recently, several case series reporting the use of adenosine to facilitate cerebral aneurysm surgery have been published, which unlike the technique described by Sollevi et al., utilize adenosine boluses rather than an infusion to produce brief periods of severe bradycardia/hypotension

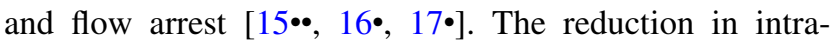
aneurysmal tension produced by an adenosine-induced flow arrest allows the surgeon to dissect the aneurysmal plane and appropriately place temporary or permanent clips.

\section{Patient Selection: Indication and Contraindication}

The decision to use adenosine-induced flow arrest during intracranial aneurysm surgery depends on a number of variables including location, size of the aneurysm, morphology and risk of rupture. In a recently published review of the use of adenosine during intracranial aneurysm surgery, Bendok et al. [17•] reported that the primary reason for using adenosine was to decrease intra-aneurysmal tension or "soften the aneurysm". Most aneurysms in this case series were located in the paraclinoid segment $(35 \%)$ or in the posterior circulation $(22.5 \%)$ [17•]. Adequate exposure of the aneurysm, its branches and perforators, is essential for safe and effective clipping of intracranial aneurysms.
This exposure is especially difficult to achieve in aneurysms that are deep or in close proximity to the skull base like the paraclinoid segment of the carotid artery and basilar apex. It is in these cases that we have found that intra-operative adenosine administration facilitates adequate exposure (see supplementary video 1). Aneurysm morphology with a broad neck [17•] and large size [18] also serve as indications to use adenosine for some neurosurgeons for similar reasons. In a recent case series, Loustarnien et al. [19] reported successful and uneventful clipping after intraoperative aneurysmal rupture facilitated by flow arrest induced by adenosine in 16 patients. The authors suggested that adenosine-induced flow arrest could be considered during intraoperative aneurysmal rupture when conventional suctioning fails to clear the field [19].

\section{Precautions}

Adenosine acts via $\mathrm{A}_{2 \mathrm{~B}}$ adenosine receptors on bronchial smooth muscles to cause muscle contraction [9]. Bebawy et al. [15••] found no significant increase in peak airway pressures following bolus administration of adenosine in patients without reactive airway disease. Although there are several case reports of bronchospasm after adenosine administration, they have always been in patients with active asthma or chronic obstructive lung disease [20, 21]. In our experience, chronic smokers without the presence of severe reactive airway disease have not had any clinically relevant bronchospasm with adenosine. Therefore, in our opinion, adenosine administration as a bolus is best avoided in patients with severe reactive airway conditions such as asthma or chronic obstructive lung disease.

In the Adenoscan multicenter trial registry, it was found that 706 out 9,250 patients $(7.6 \%)$ undergoing stress testing with adenosine developed transient atrio-ventricular (AV) conduction block [22]. Makaryus et al. [23] reported the development of a persistent AV block in patients with pre-existing right bundle branch block after large doses of adenosine (47 $\mathrm{mg}$ over $5 \mathrm{~min}$ ). Therefore, in our opinion anesthesiologists should be cautious about using adenosine in patients with pre-existing conduction defects.

Adenosine acts on the coronary vasculature to cause vasodilation, but in patients with cardiac ischemia there is a paradoxical increase in blood flow away to non-ischemic tissue (coronary vascular steal) [24, 25]. In a case report of two patients undergoing surgery for cerebral aneurysms, Owall et al. [26] reported the development of significant ST segment depression followed by sustained ventricular tachycardia in one patient and atrial flutter in the other, after administering adenosine. Both patients had a history of remote myocardial infarction ( 9 and 13 years, respectively). Bebawy et al. [15••] recommended avoidance of adenosine-induced flow arrest in patients with evidence of 
severe $(80 \%)$ left main coronary artery stenosis, or severe multi-vessel coronary artery disease (three vessels or grafts with $80 \%$ stenosis).

Finally, adenosine breakdown and uptake is inhibited by dipyridamole [13], nimodipine [27] and methylxanthines [11], leading to an increase in adenosine levels in these patients. In a study by Sollevi et al. [13], patients were administered dipyridamole intraoperatively to reduce adenosine dose requirements during cerebral aneurysm clipping. The contraindications to adenosine use are summarized in Table 1.

\section{Intra-Operative Administration of Adenosine}

The operative set up is similar to that in routine aneurysm surgery. All patients get routine intraoperative monitoring including a radial arterial line and a central venous line. We also place transcutaneous pacing pads on all our patients, as a precautionary measure, should pacing or cardioversion be required. The dose of adenosine is determined by administering escalating bolus amounts to produce a desired duration of arrest, starting with a 6 or $12 \mathrm{mg}$ initial dose (in adults). We have usually found this to be approximately $1 \mathrm{mg} / \mathrm{s}$ of arrest, i.e., severe bradycardia and hypotension, but the response can vary widely with occasional unusual sensitivity or resistance. Bebawy et al. [15••] attempted to quantify the dose-response relationship in their case series of 24 patients undergoing adenosine arrest for aneurysm surgery. They found that a median dose of $0.34 \mathrm{mg} / \mathrm{kg}$ ideal body weight (range: $0.29-0.44 \mathrm{mg} / \mathrm{kg}$ ) resulted in a systolic blood pressure of $<60 \mathrm{mmHg}$ for a median of $57 \mathrm{~s}$ (range: 26-107 s). On the basis of their results, Bebawy et al. [15••] recommended utilizing a starting dose of $0.3-0.4 \mathrm{mg} / \mathrm{kg}$ ideal body weight to achieve approximately $45 \mathrm{~s}$ of profound systemic hypotension during a remifentanil/low dose volatile anesthetic with propofol induced burst suppression. This correlates well with our experience that a dose range between 0.24 and $0.42 \mathrm{mg} / \mathrm{kg}$ is required for $30-60 \mathrm{~s}$ of hypotension [16॰]. The administration of adenosine and its subsequent repetition needs to be closely coordinated with the surgeon. Prior to repetitive dosing of adenosine, adequate time needs to elapse to ensure a return to sinus rhythm and pre-adenosine arterial pressure to avoid undesirable

Table 1 Contraindications of adenosine in cerebral aneurysm clipping

1. Severe reactive airway disease

2. Severe coronary artery disease

3. Pre-existing cardiac conduction abnormalities

4. Allergy

5. Dipyridamole, methylxanthines and nimodipine (relative contraindication) administration may prolong adenosine duration of action sequelae like hypoxic-ischemic injury or prolonged hypotension $\left[16^{\bullet}\right]$.

\section{Safety Profile}

In most patients, adequately selected for this technique, there are few if any complications during the perioperative period. Tachyphylaxis can develop in patients subjected to repeated doses of adenosine; Bebawy et al. [15••] suggested avoiding the use incremental dosing of adenosine so as to avoid tachyphylaxis. Cardiac arrest requiring chest compressions has been reported following repeat dosing of adenosine before full recovery of hemodynamic profile in one patient [16•]. Cardiac dysrhythmias like atrial fibrillation have been reported following adenosine-induced asystole [15••, 28]; in our experience, these are self-terminating and generally resolve within minutes. A large case series $(n=98)$ describing adenosine induced asystole for endovascular aortic aneurysm repair reported a $2 \%$ incidence of selflimited ST-segment depression on electrocardiography and a $4 \%$ incidence of temporary heart block requiring $<30 \mathrm{~s}$ of pacing [29]. Although the available evidence does suggest that transient ST-segment changes can be seen when adenosine is used to produce flow arrest in cerebral aneurysm surgery, there have been no reported cases of complete heart block requiring pacing or echocardiographic changes suggesting myocardial dysfunction $[15 \bullet \bullet, 16 \bullet, 17 \bullet]$. This may reflect its use in appropriately selected patients.

While there is no high level evidence comparing adenosine flow arrest assisted aneurysm clipping to the conventional technique with temporary clipping, the available literature suggests that the intraoperative use of adenosine has an acceptable safety profile and surgical experience reaffirms that it is an effective tool in optimizing aneurysm exposure and ligation. The use of rapid ventricular pacing to create intra-operative flow arrest has been recently reported and may prove a useful alternative to adenosine for intracranial aneurysm surgery [30].

\section{Conclusion}

Adenosine-assisted flow arrest in a useful technique to facilitate clip ligation of complex cerebral aneurysms. The available literature suggests that it can be used with adequate safety in carefully selected patients. The comparative efficacy of bolus adenosine to temporary clipping has not been studied, but it is clear that adenosine is a viable therapeutic option in cases where temporary clipping is high risk or technically infeasible and in cases of sudden unexpected intraoperative aneurysmal rupture. Neuroanesthesiologists should familiarize themselves with this technique and its application. 
Disclosure Shariq Khan, Shahid M. Nimjee, Nicole N. Guinn, Ali R. Zomorodi, Arthur M. Lam and Gavin W. Britz declare they have no conflict of interest. David L. McDonagh has received compensation from Cephalogics, LLC for serving as a consultant.

\section{References}

Papers of particular interest, published recently, have been highlighted as:

- Of importance

•- Of major importance

1. Schievink WI, Schaid DJ, Michels VV, Piepgras DG. Familial aneurysmal subarachnoid hemorrhage: a community-based study. J Neurosurg. 1995;83(3):426-9.

2. Wirth FP. Surgical treatment of incidental intracranial aneurysms. Clin Neurosurg. 1986;33:125-35.

3. Batjer HH, Kopitnik TA Jr, Giller CA, Samson DS. Surgical management of proximal carotid artery aneurysms. Clin Neurosurg. 1994;41:21-38.

4. Heros RC, Nelson PB, Ojemann RG, Crowell RM, DeBrun G. Large and giant paraclinoid aneurysms: surgical techniques, complications, and results. Neurosurgery. 1983;12(2):153-63.

5. Young WL, Lawton MT, Gupta DK, Hashimoto T. Anesthetic management of deep hypothermic circulatory arrest for cerebral aneurysm clipping. Anesthesiology. 2002;96(2):497-503.

6. Jafar JJ, Russell SM, Woo HH. Treatment of giant intracranial aneurysms with saphenous vein extracranial-to-intracranial bypass grafting: indications, operative technique, and results in 29 patients. Neurosurgery. 2002;51(1):138-44 discussion 144-136.

7. Fan YW, Chan KH, Lui WM, Hung KN. Retrograde suction decompression of paraclinoid aneurysm: a revised technique. Surg Neurol. 1999;51(2):129-31.

8. Bjorness TE, Greene RW. Adenosine and sleep. Curr Neuropharmacol. 2009;7(3):238-45.

9. Jacobson KA, Gao Z-G. Adenosine receptors as therapeutic targets. Nat Rev Drug Discov. 2006;5(3):247-64.

10. Honey RM, Ritchie WT, Thomson WAR. The action of adenosine upon the human heart. Quart J Med. 1930;23(92):485-9.

11. Innes JA. Review article: adenosine use in the emergency department. Emerg Med Australas. 2008;20(3):209-15.

12. Fukunaga AF, Ikeda K, Matsuda I. Atp-induced hypotensive anesthesia during surgery. Anesthesiology. 1982;57(3A):A65.

13. Sollevi A, Lagerkranser M, Irestedt L, Gordon E, Lindquist C. Controlled hypotension with adenosine in cerebral aneurysm surgery. Anesthesiology. 1984;61(4):400-5.

14. - Groff MW, Adams DC, Kahn RA, Kumbar UM, Yang BY, Bederson JB: Adenosine-induced transient asystole for management of a basilar artery aneurysm. Case report. J Neurosurg. 1999;91(4):687-690. First reported use of adenosine to arrest flow to aid clipping of a basilar tip aneurysm.

15. •• Bebawy JF, Gupta DK, Bendok BR, et al.: Adenosine-induced flow arrest to facilitate intracranial aneurysm clip ligation: doseresponse data and safety profile. Anesth Analg. 2010;110(5): 1406-1411. Large case series of adenosine assisted flow arrest to aid aneurysm clipping. The authors attempted to define a dose response profile as well as the associated compliacation with this technique.

16. • Guinn NR, McDonagh DL, Borel CO, et al.: Adenosine-induced transient asystole for intracranial aneurysm surgery: a retrospective review. J Neurosurg Anesthesiol 2011; 23(1):35-40. Large case series of adenosine assisted flow arrest to aid aneurysm clipping describing the technique in detail with associated side effects.

17. - Bendok BR, Gupta DK, Rahme RJ, et al.: Adenosine for temporary flow arrest during intracranial aneurysm surgery: a singlecenter retrospective review. Neurosurgery 2011, 69(4);815-820; discussion 820-811.Description of indications and cardiac complications associated with adenosine assisted flow arrest for aneurysm clipping.

18. Powers CJ, Wright DR, McDonagh DL, Borel CO, Zomorodi AR, Britz GW. Transient adenosine-induced asystole during the surgical treatment of anterior circulation cerebral aneurysms: technical note. Neurosurgery. 2010;67(2 Suppl Operative): 461-70.

19. Luostarinen T, Takala RS, Niemi TT, et al. Adenosine-induced cardiac arrest during intraoperative cerebral aneurysm rupture. World Neurosurg. 2010;73(2):79-83 discussion e79.

20. Jeng CS, Huang IR, Lin CP, Lin TY, Wang MJ. Intraoperative bronchospasm after intravenous adenosine during general anesthesia. Acta Anaesthesiol Taiwan. 2004;42(4):237-40.

21. DeGroff CG, Silka MJ. Bronchospasm after intravenous administration of adenosine in a patient with asthma. J Pediatr. 1994; 125(5 Pt 1):822-3.

22. Cerqueira MD, Verani MS, Schwaiger M, Heo J, Iskandrian AS. Safety profile of adenosine stress perfusion imaging: results from the adenoscan multicenter trial registry. J Am Coll Cardiol. 1994;23(2):384-9.

23. Makaryus JN, Catanzaro JN, Friedman ML, Katona KC, Makaryus AN. Persistent second-degree atrioventricular block following adenosine infusion for nuclear stress testing. J Cardiovasc Med (Hagerstown). 2008;9(3):304-7.

24. Seiler C, Kaufmann U, Meier B. Intracoronary demonstration of adenosine-induced coronary collateral steal. Heart. 1997;77(1): $78-81$.

25. Owall A, Lagerkranser M, Sollevi A. Effects of adenosineinduced hypotension on myocardial hemodynamics and metabolism during cerebral aneurysm surgery. Anesth Analg. 1988; 67(3):228-32.

26. Owall A, Gordon E, Lagerkranser M, Lindquist C, Rudehill A, Sollevi A. Clinical experience with adenosine for controlled hypotension during cerebral aneurysm surgery. Anesth Analg. 1987;66(3):229-34.

27. Blardi P, Urso R, De Lalla A, Volpi L, Perri TD, Auteri A. Nimodipine: drug pharmacokinetics and plasma adenosine levels in patients affected by cerebral ischemia. Clin Pharmacol Ther. 2002;72(5):556-61.

28. Malcolm AD, Garratt CJ, Camm AJ. The therapeutic and diagnostic cardiac electrophysiological uses of adenosine. Cardiovasc Drugs Ther. 1993;7(1):139-47.

29. Kahn RA, Moskowitz DM, Marin ML, et al. Safety and efficacy of high-dose adenosine-induced asystole during endovascular AAA repair. J Endovasc Ther. 2000;7(4):292-6.

30. Saldien V, Menovsky T, Rommens M, et al. Rapid ventricular pacing for flow arrest during cerebrovascular surgery: revival of an old concept. Neurosurgery. 2012;70(2 Suppl Operative): $270-5$. 